\title{
DETERmination OF SEPARABLe POTENTIAL From PHASE ShIfT*
}

\author{
M. BOLSTERLI \\ School of Physics, University of Minnesota, Minneapolis, \\ Minnesota, and University of California, Los Alamos \\ Scientific Laboratory, Los Alamos, New Mexico. \\ and \\ J. MacKENZIE \\ School of Physics, University of Minnesota, Minneapolis, \\ Minne sota \\ (Received 17 August 1965 )
}

\begin{abstract}
An explicit formula is given for the separable potential that fits a given phase shift. In addition, similar formulas are given for cases where there is a bound state, where sin $\delta$ changes sign, and where the phase shifts arise from coupled equations.
\end{abstract}

\section{Introduction}

IN the last few years the advantages of separable potentials [1] in calculations of three-body bound-state and scattering problems [2] and of the nuclear-matter binding energy [3] have led to their widespread use. Generally, the shape of the separable potential has been taken to be that used by Yamaguchi and Yamaguchi [1] with the addition of adjustable parameters. In this paper we show that it is possible to determine the shape of the separable potential from the two-body phase shift and binding energy. This is the analog for separable potentials of the work of Jost and Kohn and Gel' fand and Levitan [4], who showed that a local potential is determined by its two-body phase shift and bound states.

The inversion problem for local potentials requires the solution of an integral equation. For separable potentials, however, the inversion problem is much simpler, and reduces essentially to the evaluation of the Jost function, which is just a principal-value integral of the phase shift.

In Section 2 we solve the inversion problem for the simplest case. A possible bound state

* Work supported in part by the U.S. Atomic Energy Commission. 
is allowed for in Section 3. Section 4 extends the inversion to the case in which sin $\delta$ changes sign, and Section 5 gives a procedure that can be used for coupled states, as in the $n-p$ problem.

\section{Prototype}

We start with the equation for the two-body $t$ matrix in units $\hbar=2 \mu=1$, where $\mu$ is the reduced mass:

$$
t\left(\mathbf{k}, \mathbf{k}^{\prime}, E\right)=V\left(\mathbf{k}, \mathbf{k}^{\prime}\right)+\frac{1}{(2 \pi)^{3}} \int V\left(\mathbf{k}, \mathbf{k}_{1}\right) \frac{d^{3} k_{1}}{E-k_{1}^{2}} t\left(\mathbf{k}_{1}, \mathbf{k}^{\prime}, E\right)
$$

with $E$ complex. Here $V\left(\mathbf{k}, \mathbf{k}^{\prime}\right)$ is the potential in momentum space. For spinless particles, conservation of angular momentum gives

$$
\begin{gathered}
V\left(\mathbf{k}, \mathbf{k}^{\prime}\right)=\sum_{l m} V_{l}\left(k, k^{\prime}\right) Y_{l m}(\hat{k}) Y_{l m} *\left(\hat{k}^{\prime}\right) \\
t\left(\mathbf{k}, \mathbf{k}^{\prime}, E\right)=\sum_{l m} t_{l}\left(k, k^{\prime}, E\right) Y_{l m}(\hat{k}) Y_{l m} *\left(\hat{k}^{\prime}\right)
\end{gathered}
$$

and the equation for $t$ becomes

$$
t_{l}\left(k, k^{\prime}, E\right)=V_{l}\left(k, k^{\prime}\right)+\frac{1}{(2 \pi)^{3}} \int V_{l}\left(k, k_{1}\right) \frac{k_{1}^{2} d k_{1}}{E-k_{1}^{2}} t_{l}\left(k_{1}, k^{\prime}, E\right)
$$

The assumption that the potential $V_{l}\left(k, k^{\prime}\right)$ is separable is that it has the form

$$
V_{l}\left(k, k^{\prime}\right)=g_{l} v_{l}(k) v_{l}\left(k^{\prime}\right)
$$

with $g_{l}= \pm 1$. Substitution into equation (3) gives an equation that is easily solved, with the well known result (from here on the subscript $l$ is not written, but is understood to appear with all quantities)

$$
\begin{gathered}
t\left(k, k^{\prime}, E\right)=g v(k) v\left(k^{\prime}\right) / D(E), \\
D(E)=1+\frac{1}{\pi} \int_{0}^{\infty} \frac{\tau(\varepsilon)}{E-\varepsilon} d \varepsilon \\
T\left(k^{2}\right) \equiv-(4 \pi)^{-2} k g v^{2}(k) .
\end{gathered}
$$

The phase shift $\delta$ is related to $t$ by

$$
\begin{aligned}
e^{i \delta} \sin \delta & =-(4 \pi)-2 k t\left(k, k, k^{2}+i 0\right) \\
& =\tau\left(k^{2}\right) / D^{+}\left(k^{2}\right) .
\end{aligned}
$$


where

$$
D^{ \pm}\left(k^{2}\right)=\lim _{E \rightarrow k^{2} \pm i 0} \cdot D(E)
$$

In order to invert the integral equation (8) and find $\tau(E)$, we note that $D(E)$ is analytic in the cut $E$ plane and that its discontinuity across the cut is

$$
D^{+}(E)-D^{-}(E)=-2 i \tau(E), \quad E>0,
$$

so that equation (8) can be written

$$
e^{i \delta} \sin \delta=-\left(D^{+}-D^{-}\right) / 2 i D^{+},
$$

and this gives

$$
e^{2 i \delta}=D^{-} / D^{+} \text {. }
$$

A zero of $D(E)$ would correspond to a bound state in the potential. In this section we assume there is no bound state. Then it is easy to see by considering $\log D(E)$ that the solution of (12) is

$$
D(E)=e^{\tilde{\delta}}(E)
$$

where

$$
\tilde{\delta}(E) \equiv \frac{1}{\pi} \int_{-\infty}^{\infty} \frac{\delta(\varepsilon)}{E-\varepsilon} d \varepsilon
$$

is the Hilbert transform of the phase shift $\delta$, and $\delta(\varepsilon)$ has been defined to be zero for $\varepsilon<0$. We assume throughout that both $\delta(E)$ and $\tilde{\delta}(E)$ go to zero as $E$ goes to infinity.

Now by comparing (10) and (13), and using the definition (7), we find

$$
v^{2}(k)=-(4 \pi)^{2}(g / k) \text { sin } \delta\left(k^{2}\right) e^{P \tilde{\delta}\left(k^{2}\right)} .
$$

where

$$
P \tilde{\delta}(E) \equiv \frac{1}{\pi} P \int_{-\infty}^{\infty} \frac{\delta(\varepsilon)}{E-\varepsilon} d \varepsilon .
$$

Equation (15) gives the potential $v(k)$ in terms of the phase shift $\delta(E)$. An equivalent, but more cumbersome solution has been given by Gasiorowicz and Ruderman [5]. Since the exponential is positive, $g$ must be chosen so that $g$ sin $\delta$.is negative; that is, for $\delta>0$ (attractive potential), $g=-1$; for $\delta<0$ (repulsive potential), $g=+1$. Moreover, since $g$ is constant, sin $\delta$ cannot change sign; as is well known a potential of the form (4) cannot give a phase shift that goes through 0 or $\pm \pi$.

Thus, equation (15) can be used to determine a separable potential of the form (4) that will fit a two-body phase shift $\delta(E)$, provided that there is no bound state in this partial wave and that $\sin \delta(E)$ does not change sign.

In the subsequent sections we show how to include a bound state (Section 3 ), allow $\sin \delta_{l}$ 
to change sign (Section 4), and include the possibility that the particles have spin, so that the $t$ matrix equations for different $l$ values are coupled (Section 5 ).

\section{Bound state}

From equation (6) and the reality of $T(E)$ it follows that $D(E)$ can have zeros only for $E$ real. We are interested in zeros for $E \leqslant 0$. Clearly $D(E)$ can have such a zero only if $g=-1$, and it can have at most one. Let $D(E)$ have a zero at $E=-B$. Consider then

$$
D_{B}(E) \equiv D(E) /(E+B) \text {. }
$$

$D_{B}(E)$ has no zero for $E \leqslant 0$ and it follows from (12) that

$$
D_{B}-/ D_{B}{ }^{+}=e^{2 i \delta}
$$

Consider $e^{\tilde{\delta}(E)}$. This function has the appropriate discontinuity to be a solution of (18). However, since the phase shift $\delta$ goes from $\pi$ to 0 as $E$ goes from 0 to $\infty$ (Levinson's theorem holds for this nonlocal potential, since $D(E)$ has the analytic properties needed in the proof of the theorem), the function $e^{\tilde{\delta}(E)}$ has a simple zero at $E=0$ due to the jump of $\pi$ at $E=0$. Since $D_{B}$ can have no zero at $E=0$, we must use $e^{\tilde{\delta}(E)} / E$ for $D_{B}$ and therefore the appropriate solution of (12) is

$$
D(E)=(E+B) e^{\tilde{\delta}(E) / E}
$$

It is easy to see that this can also be written

$$
D(E)=e^{\tilde{\delta}_{B}(E)},
$$

where

$$
\begin{aligned}
\delta_{B}(E) & =\delta(E) & & E>0 \\
& =\pi & & -B<E<0 \\
& =0 & & E<-B
\end{aligned}
$$

Then the potential is obtained from (7) and (10) (with $g=-1$ )

$$
\begin{aligned}
v^{2}(k) & =(4 \pi)^{2}\left(k^{2}+B\right) k^{-3} \sin \delta\left(k^{2}\right) e^{P \delta \tilde{\delta}\left(k^{2}\right)} \\
& =(4 \pi)^{2} k^{-1} \sin \delta\left(k^{2}\right) e^{P \tilde{\delta}_{B}\left(k^{2}\right)} .
\end{aligned}
$$

where the second form is more convenient for computation. 


\section{4. sin $\delta$ changes sign}

The nice feature of the separable potential of the two preceding sections is that an explicit formula can be given for computing the potential from the phase shift and the location of the bound state. A certain amount of flexibility has had to be sacrificed, in that the range of $\sin \delta$ and the number of bound states have had to be restricted. In this section we show how to remove these restrictions in such a way as to retain the essential feature, namely, the explicit formula for the potential in terms of the phase shift.

Suppose that $\sin \delta$ changes sign at $E=\varepsilon_{0}$. Then it is easy to see that the phase shift can be fitted with a separable potential of the form

$$
\begin{aligned}
V\left(k, k^{\prime}\right) & =g_{1} v(k) v\left(k^{\prime}\right) & k^{2}<\varepsilon_{0}, & k^{\prime 2}<\varepsilon_{0}, \\
& =g_{2} v(k) v\left(k^{\prime}\right) & k^{2}>\varepsilon_{0}, & k^{\prime 2}>\varepsilon_{0} . \\
& =0 & & \text { otherwise. }
\end{aligned}
$$

However, this potential gives a $t$ matrix which is zero in the regions where $V$ vanishes, and therefore seems very unlike the $t$ matrix that would be obtained from a local potential.

We have preferred to use another separable potential, of the form

$$
\begin{aligned}
V\left(k, k^{\prime}\right) & =g v(k) v\left(k^{\prime}\right) \text { if } k^{2}<\varepsilon_{0} \text { or } k^{\prime 2}<\varepsilon_{0} . \\
& =\gamma g v(k) v\left(k^{\prime}\right) \text { if } k^{2}>\varepsilon_{0} \text { and } k^{\prime 2}>\varepsilon_{0}
\end{aligned}
$$

with $g= \pm 1$ and $\gamma$ arbitrary.

Again the equation for $t$ can be solved, and the on-the-energy-shell equations take the forms (11) and (12), with $D(E)$ now given by

$$
\begin{gathered}
D(E)=1+\gamma \tilde{\tau}_{>}(E)+\tilde{\tau}_{<}(E)-(1-\gamma) \tilde{\tau}_{>}(E) \tilde{\tau}_{<}(E) \\
=(1-\gamma)-1-(1-\gamma)\left(\tilde{\tau}_{<}(E)-\gamma /(1-\gamma)\right)\left(\tilde{\tau}_{>}(E)-(1-\gamma)-1\right), \\
{ }^{\top}>(E) \equiv \tau(E) \theta\left(E-\varepsilon_{0}\right), \\
{ }^{\top}(E) \equiv \tau(E) \theta\left(\varepsilon_{0}-E\right),
\end{gathered}
$$

and $T(E)$ again given by (7). It follows as before that

$$
D(E)=e^{\tilde{\delta}(E)}
$$

We discuss only the case $g=-1$ in the following. Then it follows from (25) that $D(E)$ can have at most one zero for $E \leqslant 0$. This can be included in (27) by using the methods of the 
previous section. Here we consider only the case that $D(E)$ has no zero. Now let

$$
F(E)=D(E)-(1-\gamma)^{-1}
$$

If we choose $\gamma$ so that $(1-\gamma)^{-1}<D(0)$, then $F(E)$ also has no zeros for $E \leqslant 0$. Therefore, if we define $\xi$ by

$$
e^{2 i \xi} \equiv F^{-}(E) / F^{+}(E)
$$

it follows that

$$
\xi(0)-\xi(\infty)=0
$$

by the same methods as those used in proving Levinson's theorem.

Now since ${ }_{>}(E)$ is real for $E<\varepsilon_{0}$, it follows that

$$
e^{2 i \xi}=\frac{\left(\tilde{\tau}_{<}^{-}-\gamma(1-\gamma)^{-1}\right)}{\left(\tilde{\tau}^{+}-\gamma(1-\gamma)^{-1}\right)}, \quad E<\varepsilon_{0},
$$

and therefore

$$
\tilde{\tau}_{<}-\gamma(1-\gamma)^{-1}=-\gamma(1-\gamma)^{-1} e^{\tilde{\xi}_{<}} .
$$

where

$$
\xi_{<}(E)=\xi(E) \theta\left(\varepsilon_{0}-E\right) .
$$

Similarly

$$
\tilde{\tau}_{>}-(1-\gamma)-1=-(1-\gamma)-1 e^{\tilde{\xi}_{>}}
$$

with

$$
\xi_{>}(E)=\xi(E) \theta\left(E-\varepsilon_{0}\right) .
$$

It follows that

$$
\begin{aligned}
\tau(E) & =-\gamma(1-\gamma)-1 \sin \xi e^{P \tilde{\xi}_{<}} \quad E<\varepsilon_{0} . \\
& =-(1-\gamma)-1 \sin \xi e^{P \tilde{\xi}_{>}} \quad E>\varepsilon_{0} .
\end{aligned}
$$

so that $\tau$ will not change sign if only $\gamma$ is chosen to be negative. The only other condition on $\gamma$ for the case we have considered is

$$
\left(1-\gamma^{-1}<D(0)\right. \text {. }
$$

Any negative $\gamma$ satisfying (37) can be used to obtain a $\xi$ from (28) and (29), and, hence, $\tau(E)$ from (36). 


\section{Coupled equations}

We consider the case of spin-1/2 particles. Then in place of (2), we must use the resolutions

$$
\begin{aligned}
V\left(\mathbf{k}, \mathbf{k}^{\prime}\right) & =\sum_{J S L L^{\prime} M} V_{L L}, S J\left(k, k^{\prime}\right) \mathscr{Y}_{M} S L J(\hat{k}, \sigma) \mathscr{Y}_{M} S L^{\prime} J^{*}(\hat{k}, \sigma), \\
t\left(\mathbf{k}, \mathbf{k}^{\prime}, E\right) & =\sum_{J S L L^{\prime} M} t_{L L}, S J\left(k, k^{\prime}, E\right) \mathscr{Y}_{M} S L J(\hat{k}, \sigma) \mathscr{Y}_{M^{\prime}} S L^{\prime} J^{*}\left(\hat{k}^{\prime}, \sigma\right),
\end{aligned}
$$

where the $\mathscr{Y}_{M} S L J$ are the vector spherical harmonics for two spin-1/2 particles [6]. Then the equation for $t$ becomes

$$
\begin{gathered}
t_{L L}{ }^{\prime S J}\left(k, k^{\prime}, E\right)=V_{L L}{ }^{\prime S J}\left(k, k^{\prime}\right)+ \\
\frac{1}{(2 \pi)^{3}} \sum_{L^{\prime \prime}} \int V_{L L^{\prime \prime}} S J\left(k, k^{\prime \prime}\right) \frac{k^{\prime \prime 2} d k^{\prime \prime}}{E-k^{\prime \prime 2}} t_{L^{\prime \prime} L^{\prime}} S J\left(k, k^{\prime}, E\right)
\end{gathered}
$$

For $S=0$, only $L=L^{\prime}$ is allowed; there are no coupled equations and the previous sections apply. Similarly, for $S=1$ and parity $(-)^{J}$, there is again a single equation. Only in the case $S=1$ and parity $(-)^{J+1}$ are the equations for $L=J+1$ and $L=J-1$ coupled. In this case it is well known that on the energy shell $t$ takes the form

$$
t_{L L}, S J\left(k, k, k^{2}+i 0\right)=\sum_{\eta=\alpha, \beta} M_{L \eta} S J t_{\eta} S J \tilde{M}_{\eta L}, S J \text {, }
$$

where the orthogonal matrix $M$ is

$$
M_{L \eta} S J=\left(\frac{\cos \varepsilon_{J}}{\sin \varepsilon_{J}}-\frac{\sin \varepsilon_{J}}{\cos \varepsilon_{J}}\right)
$$

and

$$
t_{\eta} S J=-\frac{(4 \pi)^{2}}{k} e^{i \delta_{\eta} J} \sin \delta_{\eta}^{J}
$$

The $t$ matrix is characterized by the two phase shifts $\delta_{\alpha}$ and $\delta_{\beta}$ and the mixing parameter $\varepsilon_{J}$.

It is therefore convenient to define 


$$
\begin{aligned}
& t_{\eta \zeta^{\prime}} S J\left(k, k^{\prime}, E\right)=\sum_{L L^{\prime}} \tilde{M}_{\eta L^{\prime}} S J^{\left(k^{2}\right)} t_{L L^{\prime}} S J\left(k, k^{\prime}, E\right) M_{L} \zeta^{S J}\left(k^{\prime 2}\right)
\end{aligned}
$$

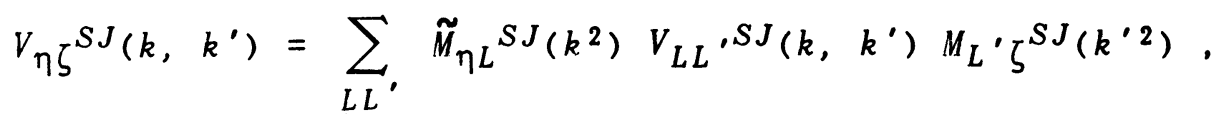

so that the equation for $t$ becomes

$$
\begin{aligned}
& t_{\eta \zeta} S J\left(k, k^{\prime}, E\right)=V_{\eta \zeta} S J\left(k, k^{\prime}\right)+
\end{aligned}
$$

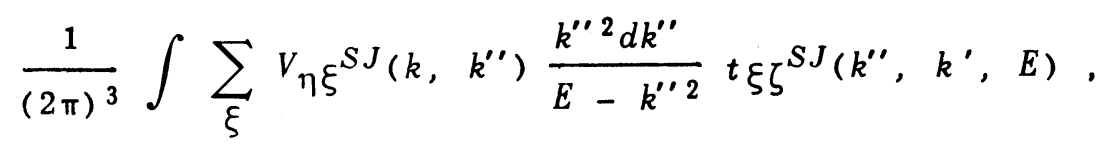

where it follows from (43) that $t_{\eta} S J$ is diagonal on the energy shell:

$$
t_{\eta \zeta} S J\left(k, k, k^{2}+i 0\right)=-\delta_{\eta \zeta} \frac{4 \pi^{2}}{k} e^{i \delta_{\eta} \sin \delta_{\eta} .}
$$

In order to have simple equations, we continue off the energy shell in the simplest possible way, namely, we assume that $t_{\eta \zeta}$ and $V_{\eta \zeta}$ are diagonal everywhere. This gives the uncoupled equations

$$
\begin{gathered}
t_{\eta} S J\left(k, k^{\prime}, E\right)=V_{\eta} S J\left(k, k^{\prime}\right)+ \\
\frac{1}{(2 \pi)^{3}} \int V_{\eta} S J\left(k, k^{\prime \prime}\right) \frac{k^{\prime \prime 2} d k^{\prime \prime}}{E-k^{\prime \prime 2}} t_{\eta} S J\left(k^{\prime \prime}, k^{\prime}, E\right)
\end{gathered}
$$

which can be solved by the methods of the previous sections if the potential $V_{\eta} S J\left(k, k^{\prime}\right)$ is assumed to have the form postulated there. For example, if $\sin \delta_{\alpha}$ has no zero, we take

$$
V_{\alpha} S J\left(k, k^{\prime}\right)=g_{\alpha} S J \quad v_{\alpha} S J(k) v_{\alpha} S J\left(k^{\prime}\right)
$$

and find

$$
g_{\alpha} S J v_{\alpha} S J(k)^{2}=(4 \pi)^{2}\left(g_{\alpha} S J / k\right) \sin \delta_{\alpha} S J e^{\frac{1}{\pi} f^{\infty} \frac{\delta_{\alpha} S J(\varepsilon)}{k^{2}-\varepsilon} d \varepsilon}
$$

The mixing reappears when we transform back to the $L L^{\prime}$ representation:

$$
V_{L L}, S J\left(k, k^{\prime}\right)=\sum_{\eta} M_{L \eta} S J\left(k^{2}\right) V_{\eta} S J\left(k, k^{\prime}\right) \tilde{M}_{\eta L}{ }^{\prime S J}\left(k^{\prime}{ }^{2}\right)
$$




\section{Summary}

The preceding sections give formulas for finding a separable potential to fit a given phase shift. Of course, in practice the phase shift is only available up to a certain energy, so that beyond that energy the phase shift must be guessed. A subsequent paper will describe the effects of different high-energy phase shifts on the form of the separable potential.

\section{References}

1. Y. YAMAGUCHI and Y. YAMAGUCHI, Phys. Rev. 95, 1628 and 1635 (1954); A.N. MITRA and V.L. NARASIMHAM, Nucl. Phys. 14, 407 (1960).

2. For example, R. AARON, R.D. AMADO and Y.Y. YAM, Phys. Rev. 136, B650 (1964); M. BANDER, Phys. Rev. 138, B322 (1965); J.H. HETHERINGTON and L.H. SCHICK, Phys. Rev. 138, B1411 (1965).

3. For example, F. TABAKIN, Ann. Phys. (N.Y.) 30, 51 (1964); C. B. DUKE, Phys. Rev. 136, B49 (1964).

4. The review article by R.G. NEWTON, J. Math. Phys. 1, 319 (1960) gives references to the original papers.

5. S. GASIOROWICZ and M.A. RUDERMAN, Phys. Rev. 110, 261 (1958).

6. J.M. BLATT and V.F. WEISSKOPF, Theoretical Nuclear Physics, John Wiley, New York (1952). 